\title{
Fast Algorithms for Displacement and Low-Rank Structured Matrices
}

\author{
Shivkumar Chandrasekaran \\ University of California Santa \\ Barbara \\ Santa Barbara, California \\ shiv@ucsb.edu
}

\author{
Nithin Govindarajan \\ University of California Santa \\ Barbara \\ Santa Barbara, California \\ ngovindarajan@ucsb.edu
}

\author{
Abhejit Rajagopal \\ University of California Santa \\ Barbara \\ Santa Barbara, California \\ abhejit@ucsb.edu
}

\begin{abstract}
This tutorial provides an introduction to the development of fast matrix algorithms based on the notions of displacement and various low-rank structures.
\end{abstract}

\section{KEYWORDS}

Fast matrix algorithms, displacement structure, low-rank, sequentially semi-separable, hierarchically semi-separable, Toeplitz, Hankel, Vandermonde, Cauchy, Hilbert, Fast multipole method

\section{ACM Reference Format:}

Shivkumar Chandrasekaran, Nithin Govindarajan, and Abhejit Rajagopal. 2018. Fast Algorithms for Displacement and Low-Rank Structured Matrices. In ISSAC '18: 2018 ACM International Symposium on Symbolic and Algebraic Computation, fuly 16-19, 2018, New York, NY, USA. ACM, New York, NY, USA, 7 pages. https://doi.org/10.1145/3208976.3209025

\section{INTRODUCTION}

In this tutorial we give a broad introduction to the class of displacement structured and other low-rank structured matrices that have come to recent prominence. Due to page limitations this paper only provides a short summary of the actual topics that will be explained in the tutorial itself.

As is well-known, it costs roughly $O\left(n^{3}\right)$ arithmetic operations to multiply two $n \times n$ generic matrices (as long as no Strassen-style fast multiplication algorithm is used ${ }^{1}$ ). Similarly, the solution to a generic linear system of of $n$ equations in $n$ unknowns requires $O\left(n^{3}\right)$ operations (again assuming we do not use a fast matrix multiplication algorithm).

However, when we consider a structured family of matrices it might be possible to find practically fast algorithms. Famous examples include the FFT method [10] for multiplying the discrete Fourier series matrix rapidly. As a fallout of the FFT, we get a fast method for convolution and as a result a large number of fast algorithms for polynomials [14], which in turn yield fast algorithms for Toeplitz matrices. However, many of these fast algorithms did

\footnotetext{
${ }^{1}$ We discard Strassen style algorithms as they are slower than the standard algorithm for reasonable values of $n$.

Permission to make digital or hard copies of all or part of this work for personal or classroom use is granted without fee provided that copies are not made or distributed for profit or commercial advantage and that copies bear this notice and the full citation on the first page. Copyrights for components of this work owned by others than the author(s) must be honored. Abstracting with credit is permitted. To copy otherwise, or republish, to post on servers or to redistribute to lists, requires prior specific permission and/or a fee. Request permissions from permissions@acm.org. ISSAC '18, fuly 16-19, 2018, New York, NY, USA

(c) 2018 Copyright held by the owner/author(s). Publication rights licensed to ACM. ACM ISBN 978-1-4503-5550-6/18/07 . \$15.00

https://doi.org/10.1145/3208976.3209025
}

not prove so useful in practice [17]. Some of them, were too slow for reasonable values of $n$, whereas others required large number of bits to hold intermediate values.

Eventually some of these shortcomings were over come by exploiting low-rank structures that are lurking in these problems (e.g. $[6,8,30])$. In this tutorial we focus on two aspects of this. The first is the displacement structure approach pioneered by Kailath et. al. [21, 22]. The second is the low-rank structured approach pioneered by Rokhlin [18, 26, 27], Hackbusch [19], Eidelman and Gohberg [13], Dewilde and van der Veen [11], and others.

\section{DISPLACEMENT STRUCTURE}

We eschew generality in this presentation and also do not worry about superfast algorithms. For simplicity we restrict ourselves to the real field.

Let the linear operator $\mathcal{L}[A, B]: \mathcal{R}^{n \times n} \rightarrow \mathcal{R}^{n \times n}$ be defined as

$$
\mathcal{L}[A, B](T)=T-A T B^{T},
$$

where $A, B \in \mathcal{R}^{n \times n}$. We will call such a linear operator as a displacement operator. We say that the matrix $T$ has a displacement structure (wrt $\mathcal{L}[A, B]$ ) if the rank of $\mathcal{L}[A, B](T)$ is small compared to $n$. The standard examples are Toeplitz, Hankel, Vandermonde, Cauchy and Pick matrices.

- Let $Z$ denote the down-shift matrix

$$
Z_{i, j}= \begin{cases}0, & i \neq j-1 \\ 1, & i=j-1 .\end{cases}
$$

Then it is easy to check that if $T$ is a Toeplitz matrix rank of $\mathcal{L}[Z, Z](T)$ is at most 2 . Conversely, any $T$ for which rank of $\mathcal{L}[Z, Z]$ is small is called a Toeplitz-like matrix.

- Let $x_{i} \in \mathcal{R}$ and let $D(x)=\operatorname{diag}\left\{x_{i}\right\}$. Let $V$ be the Vandermonde matrix: $V_{i, j}=x_{i}^{j}$. Then rank of $\mathcal{L}[D(x), Z](V)$ is at most 1 .

- Let $0 \neq y_{j} \in \mathcal{R}$. Let $C$ be the Cauchy matrix: $C_{i, j}=1 /\left(y_{i}-\right.$ $x_{j}$ ). Then rank of $\mathcal{L}\left[D^{-1}(y), D(x)\right](C)$ is at most 1 . Conversely any matrix $C$ for which rank of $\mathcal{L}[D(y), D(x)](C)$ is small is called a generalized Pick matrix.

What is surprising is that the inverse has the same structure too. In fact

$$
\operatorname{rank}(\mathcal{L}[A, B](T))=\operatorname{rank}\left(\mathcal{L}[B, A]\left(T^{-1}\right)\right) .
$$

More trivially

$$
\begin{aligned}
\operatorname{rank}\left(\mathcal{L}[A, B]\left(T_{1}+T_{2}\right)\right) \leq & \operatorname{rank}\left(\mathcal{L}[A, B]\left(T_{1}\right)\right)+ \\
& \operatorname{rank}\left(\mathcal{L}[A, B]\left(T_{2}\right)\right) .
\end{aligned}
$$


Unfortunately the result for products is not as nice:

$$
\begin{gathered}
\operatorname{rank}\left(\mathcal{L}[A, B]\left(T_{1} T_{2}\right)\right) \leq \operatorname{rank}\left(\mathcal{L}[A, B]\left(T_{1}\right)\right)+ \\
\operatorname{rank}\left(\mathcal{L}[A, B]\left(T_{2}\right)\right)+\operatorname{rank}\left(B^{T} A-I\right) .
\end{gathered}
$$

In particular this is rather disastrous for generalized Pick (Cauchy) matrices, which will be rectified later.

We next look for fast algorithms that can exploit the displacement structure. Suppose that

$$
T-A T B^{T}=P Q^{T},
$$

where $P, Q \in \mathcal{R}^{n \times p}$. If $\mathcal{L}[A, B]$ is invertible then the pair $(P, Q)$ can be used as a more efficient representation of the matrix $T$ as we would require only $O(n p)$ numbers versus the usual $O\left(n^{2}\right)$ numbers. In this case the pair $(P, Q)$ will be called generators for $T$. The question is: can we carry out the usual matrix operations faster using the pair $(P, Q)$ ? Surprisingly, this question is easier to answer for $T^{-1} x$ rather than for $T x$, for $x \in \mathcal{R}^{n}$. In particular we will show that the $L U$ factorization (Gaussian elimination) of $T$ can be computed quickly in $O\left(n^{2}\right)$ arithmetic operations rather than the standard $O\left(n^{3}\right)$ operations, provided inverting $\mathcal{L}[A, B]$ itself is cheap.

We note that the displacement rank is invariant under similarity transformations

$$
\operatorname{rank}\left(\mathcal{L}\left[V A V^{-1}, W B W^{-1}\right]\left(V T W^{T}\right)\right)=\operatorname{rank}(\mathcal{L}[A, B](T)) .
$$

Therefore it is convenient to assume that $A$ and $B$ are lower triangular matrices from now on (say using the Schur decomposition). Note that for this to be effective in fast algorithms, the computation and application of $V, V^{-1} W$ and $W^{-1}$, must cost less than $O\left(n^{3}\right)$ operations. There are extensions of the method to the case when $A$ or $B$ is a lower Hessenberg matrix [20], but we do not cover it here.

Let $\mathcal{L}[A, B](T)$ have low-rank with $A$ and $B$ lower triangular and let

$$
T={ }_{n-1}^{1}\left(\begin{array}{cc}
1 & n-1 \\
T_{11} & T_{12} \\
T_{21} & T_{22}
\end{array}\right), \quad S=T_{22}-T_{21} T_{11}^{-1} T_{12},
$$

where $S$ is the Schur complement of $T$ and we have have assumed that $T_{11} \neq 0$. The key idea of fast Gaussian elimination is that $S$ has a low-rank displacement structure that can be quickly computed from that of $T$. To see this, let

$$
A={ }_{n-1}^{1}\left(\begin{array}{cc}
1 & n-1 \\
A_{11} & 0 \\
A_{21} & A_{22}
\end{array}\right), \quad B={ }_{n-1}^{1}\left(\begin{array}{cc}
1 & n-1 \\
B_{11} & 0 \\
B_{21} & B_{22}
\end{array}\right) .
$$

Then it can be readily shown that rank of $\mathcal{L}\left[A_{22}, B_{22}\right](S)$ is at most rank of $\mathcal{L}[A, B](T)$, and furthermore the generators for $S$ can be computed in $O\left(n p^{2}\right)$ operations provided that the first column and row of $T$ can be generated in $O(n)$ operations ${ }^{2}$ from its generators $(P, Q)$. The recursive application of this idea leads to the fast generalized Schur algorithm for computing the $L U$ factorization of $T$ in $O\left(n^{2}\right)$ operations.

For many applications already this speed-up is sufficient. However there is sometimes a need for more. For example if $T$ is a

\footnotetext{
${ }^{2}$ This requires that $A$ and $B$ be specially structured lower-triangular matrices. The shift-down and diagonal matrices are good examples.
}

Toeplitz matrix we can compute $T x$ in $O\left(n \log _{2} n\right)$ operations using the FFT. The Gohberg-Semencul formulas $[9,16]$ show that there is a representation for $T^{-1}$ (which might require some time to compute) such that $T^{-1} x$ can also be computed in $O\left(n \log _{2} n\right)$ operations via the FFT. The displacement structure approach can also shed light on this situation.

First note that $T^{-1}$ is a Schur complement of

$$
M=\left[\begin{array}{cc}
T & I \\
-I & 0
\end{array}\right]
$$

So we could compute the generators of $T^{-1}$ by running the generalized Schur algorithm on $M$ half-way through. This would be fast if $M$ had a short displacement rank for an appropriate $\mathcal{L}\left[A_{M}, B_{M}\right]$. One potential choice is

$$
A_{M}=\left[\begin{array}{ll}
A & \\
& A
\end{array}\right], \quad B_{M}=\left[\begin{array}{ll}
B & \\
& B
\end{array}\right],
$$

provided rank of $I-A B^{T}$ is small. Another possibility is

$$
A_{M}=\left[\begin{array}{cc}
A & \\
& B^{T \dagger}
\end{array}\right], \quad B_{M}=\left[\begin{array}{ll}
B & \\
& A^{T \dagger}
\end{array}\right],
$$

provided the orthogonal projectors $I-B B^{\dagger}$ and $I-A A^{\dagger}$ have small rank.

For generalized Pick matrices $P$ where the rank of $\mathcal{L}\left[D_{1}, D_{2}\right](P)$ is small, with $D_{i}$ a diagonal matrix, we have a problem if $\mathcal{L}\left[D_{1}, D_{2}\right]$ is not invertible. In this case we can still make progress via a rank-1 perturbation of the form $\mathcal{L}\left[D_{1}+u u^{T}, D_{2}\right](P)$ for a well-chosen column $u$, and exploiting the fact the eigenvector matrix of $D_{1}+u u^{T}$ is an orthogonal Cauchy matrix, which can be multiplied rapidly using one of several techniques. This rank 1 perturbation will increase the displacement rank of $P$ by at most 1 so the fast generalized Schur algorithm can still be deployed.

Once the generators for $T^{-1}$ have been computed, the question naturally arises as to how to multiply $T^{-1} x$ quickly. If $|\lambda(A)|<1$ and $|\lambda(B)|<1$, then we have the series solution for $\mathcal{L}[A, B](T)=$ $P Q^{T}$ as:

$$
T=\sum_{l=0}^{\infty}\left(A^{l} P\right)\left(B^{l} Q\right)^{T} .
$$

If $T$ is the inverse of a Toeplitz matrix and $A=B=Z$, then this gives the celebrated Gohberg-Semencul formula as $A$ and $B$ are nilpotent. When $A$ and $B$ are diagonal then $T$ is a generalized Cauchy (Pick) matrix and there are fast algorithms for matrixvector multiply [15]. Some generalizations are available in [1]. However there seems to be no general approach for multiplying an arbitrary low displacement-rank matrix with a vector for a general class of nice $A$ and $B$.

We do make the general observation that if the sum (1) is converging rapidly then one can easily construct a fast approximate matrix-vector multiplication algorithm. But those matters are better dealt with later in this tutorial.

It is easy to see how to compute the generators for $T_{1}+T_{2}$ quickly from those of $T_{i}$ provided they have low displacement-rank for the same displacement operators $\mathcal{L}[A, B]$. Similarly it is not difficult to quickly compute the generators of $T_{1} T_{2}$ provided that we can rapidly multiply by both $A$ and $B$, and that a low rank factorization of $I-B^{T} A$ is quickly available. 
The big draw back of displacement structure approach is that there has been no progress on generalizing it to nested structures like Toeplitz-block-Toeplitz matrices. This might in turn be related to the fact that the inverses of higher-order Sylvester-Stein operators are not well-understood.

\section{SEQUENTIALLY SEMI-SEPARABLE (SSS) REPRESENTATIONS}

Just like displacement structure theory was born from systems theory (study of linear time-invariant [LTI] systems), another study of low-rank structured matrices was born from trying to generalize systems theory to time-varying systems [11]. We do not attempt a generalized approach in this tutorial. (More information can be found in $[3,6,7]$.)

For any square matrix $A$ consider partitions of the form

$$
A={ }_{n-m}^{m}\left(\begin{array}{cc}
m & n-m \\
A_{11} & A_{12} \\
A_{21} & A_{22}
\end{array}\right) .
$$

We will call such off-diagonal blocks as $A_{12}$ and $A_{21}$ as Hankel blocks. Note that these off-diagonal blocks never cross the principal diagonal and always extend as far to the corners as possible. In this section we study families of matrices for which the ranks of all Hankel blocks are small compared to the matrix size $n$. In particular it turns out that there is a non-linear representation of the matrix that captures this structure precisely and permits a full spectrum of fast linear (in matrix size $n$ ) algorithms.

The key question is what constraints are placed on the matrix entries by the requirement that two overlapping Hankel blocks must have low rank? Consider the following partition of $A$ :

$$
A=\left[\begin{array}{lll}
A_{11} & A_{12} & A_{13} \\
A_{21} & A_{22} & A_{23} \\
A_{31} & A_{32} & A_{33}
\end{array}\right]
$$

where both $\mathcal{H}_{1}=\left[\begin{array}{ll}A_{12} & A_{13}\end{array}\right]$ and $\mathcal{H}_{2}=\left[\begin{array}{ll}A_{13}^{T} & A_{23}^{T}\end{array}\right]^{T}$ are Hankel blocks. From the presence of the shared common block $A_{13}$ we see that the column space of $\mathcal{H}_{1}$ must be related to the column space of $\mathcal{H}_{2}$, and similarly the row-space of $\mathcal{H}_{2}$ must be related to the row-space of $\mathcal{H}_{1}$. To that end, let:

$$
\mathcal{H}_{1}=U_{1}\left[\begin{array}{ll}
V_{11}^{T} & V_{12}^{T}
\end{array}\right], \quad \mathcal{H}_{2}=\left[\begin{array}{c}
U_{21} \\
U_{22}
\end{array}\right] V_{2}^{T},
$$

be full column-rank factorizations of $\mathcal{H}_{i}$ conformally partitioned with $A_{13}=U_{1} V_{12}^{T}=U_{21} V_{2}^{T}$. For a fixed choice of the two factorizations there is a unique matrix $W$, which we call a transition operator, such that

$$
U_{21}=U_{1} W, \quad V_{12}^{T}=W V_{2}^{T} .
$$

If the rank of $\mathcal{H}_{i}$ is $r_{i}$ then $W \in \mathcal{R}^{r_{1} \times r_{2}}$.

If we now choose $\sum_{i=1}^{p} n_{i}=n$, and use it to partition $A$ into a block $p \times p$ matrix, such that the sub-block $A_{i j} \in \mathcal{R}^{n_{i} \times n_{j}}$, then we can use the above idea repeatedly on adjacent Hankel blocks to construct a representation of the blocks of $A$ of the following form:

$$
A_{i j}= \begin{cases}D_{i}, & i=j, \\ U_{i} W_{i+1} W_{i+2} \cdots W_{j-1} V_{j}^{T}, & i<j, \\ P_{i} R_{i-1} R_{i-2} \cdots R_{j+1} Q_{j}^{T}, & i>j .\end{cases}
$$

A simple example is a banded matrix which clearly has the desired low-rank property for the Hankel blocks. In this case if we choose $n_{i}$ to be equal to the band-width we can write down the components directly.

$$
\begin{aligned}
D_{i} & =A_{i, i} \\
U_{i} & =A_{i, i+1} \\
V_{i} & =I \\
P_{i} & =A_{i, i-1} \\
Q_{i} & =I \\
R_{i} & =0 \\
W_{i} & =0 .
\end{aligned}
$$

To understand this representation better we look at how you can compute $A x=b$ fast. A little bit of algebra reveals that the following recursions will do the job:

$$
\begin{aligned}
g_{i} & =V_{i}^{T} x_{i}+W_{i} g_{i+1} \\
h_{i} & =Q_{i}^{T} x_{i}+R_{i} h_{i-1} \\
b_{i} & =D_{i} x_{i}+U_{i} g_{i+1}+P_{i} h_{i-1},
\end{aligned}
$$

where all undefined variables are assumed to be empty matrices of suitable size, and $x_{i}$ and $b_{i}$ are conformal partitions of $x$ and $b$ respectively. It is clear that if all Hankel block ranks are small compared with the matrix size then this is a linear time algorithm for computing $A x$.

However there are more to these recursions. We make the notation, for example, that

$$
D=\left[\begin{array}{cccc}
D_{1} & & & \\
& D_{2} & & \\
& & \ddots & \\
& & & D_{p}
\end{array}\right] \quad g=\left[\begin{array}{c}
g_{1} \\
g_{2} \\
\vdots \\
g_{p}
\end{array}\right] .
$$

The recursions can then be written in matrix form as

$$
\left[\begin{array}{ccc}
I-W Z^{T} & 0 & -V^{T} \\
0 & I-R Z & -Q^{T} \\
U Z^{T} & P Z & D
\end{array}\right]\left[\begin{array}{l}
g \\
h \\
x
\end{array}\right]=\left[\begin{array}{l}
0 \\
0 \\
b
\end{array}\right] .
$$

From this we get the diagonal representation of $A$ as

$$
A=D+U Z^{T}\left(I-W Z^{T}\right)^{-1} V^{T}+P Z(I-R Z)^{-1} Q^{T} .
$$

In other words $A$ is just the Schur complement of a large sparse matrix. In particular we will call $(D, U, W, V, P, R, Q)$ as the SSS representation of $A$.

Furthermore there is a re-ordering of the unknowns in equation (3) into the sequence $\left(g_{i}, h_{i}, x_{i}\right)$, and if the rows of the sparse matrix in (3) are also re-ordered the same way we can then see that the the sparse matrix will be a linear graph by referring to the original recursions in (2). Therefore it follows that in this ordering the sparse matrix has a no fill-in elimination order and that therefore there is a fast algorithm (sparse Gaussian elimination) to quickly compute $x$ (and incidentally also $g$ and $h$ ) from $b$. Some elementary calculations then reveal that the resulting algorithm wll be linear in the matrix size $n$.

However more operations involving SSS representations can be done in linear time. The key to understanding this is the simple 
fact that low-rank Hankel blocks imply short SSS representations and vice versa.

For example, since

$$
\left[\begin{array}{ll}
A_{11} & A_{12} \\
A_{21} & A_{22}
\end{array}\right]\left[\begin{array}{ll}
B_{11} & B_{12} \\
B_{21} & B_{22}
\end{array}\right]=\left[\begin{array}{cc}
* & A_{11} B_{12}+A_{12} B_{22} \\
* & *
\end{array}\right]
$$

it follows that the product of two SSS matrices will be another SSS matrix whose Hankel block ranks are at most the sum of the corresponding Hankel blocks of the original SSS matrices. With some effort a linear time algorithm can be constructed for producing the SSS representation of $A B$ given those of $A$ and $B$.

Similarly from

$$
\left[\begin{array}{ll}
A_{11} & A_{12} \\
A_{21} & A_{22}
\end{array}\right]^{-1}=\left[\begin{array}{cc}
* & * A_{12} * \\
* A_{21} * & *
\end{array}\right],
$$

where the *'s denote matrices that we do not care about, it follows that the Hankel block ranks of the inverse are at most those of the original matrix. It therefore follows, for example, that both banded matrices and their inverse have short SSS representations. Though not all matrices with short SSS representations are the inverses of banded matrices, the diagonal representation (4) shows that every SSS representation is the Schur complement of a larger banded matrix in the right ordering.

One way to quickly compute the SSS representation of the inverse is as follows. First we observe that since

$$
\left[\begin{array}{ll}
A_{11} & A_{12} \\
A_{21} & A_{22}
\end{array}\right]=\left[\begin{array}{cc}
I & 0 \\
A_{21} A_{11}^{-1} & I
\end{array}\right]\left[\begin{array}{cc}
A_{11} & A_{12} \\
0 & A_{22}-A_{21} A_{11}^{-1} A_{12}
\end{array}\right],
$$

both $L$ and $U$ in the $L U$ factorization of $A$ will have exactly the same Hankel block ranks as $A$. From this with some algebra one can construct a linear time algorithm to compute the SSS representations of $L$ and $U$ from that of $A$. Then, using the diagonal representation and the Woodbury formula, we can compute in linear time the SSS representation of $L^{-1}$ and $U^{-1}$. We can then use the aforementioned linear time multiplication algorithm to find the SSS representation of $U^{-1} L^{-1}=A^{-1}$.

We also note that there are linear time algorithms for the $U L V$ factorization of $A$ in SSS form, if growth factor becomes an issue.

Clearly the sum of two SSS matrices is another SSS matrix. So effectively matrix algebra is fast in the SSS representation and this has proven to be tremendously useful in practice.

\section{HIERARCHICALLY SEMI-SEPARABLE (HSS) REPRESENTATIONS}

There is another non-linear matrix representation that is also capable of providing a linear time matrix algebra. This was born independently from efforts to speed up the application of integral operators that arise in potential theory in the fundamental work of Greengard and Rokhlin on the fast multi-pole method (FMM). The representation we present now (HSS) is a special case of this more general class of FMM representations. The more general class is not closed under inversion and multiplication, but the HSS representation is. More detailed information can be found in [2, 5, 12, 23$25,28,29]$.
The HSS representation uses a slightly different definition of Hankel blocks. Let

$$
A=\left[\begin{array}{lll}
A_{11} & A_{12} & A_{13} \\
A_{21} & A_{22} & A_{23} \\
A_{31} & A_{32} & A_{33}
\end{array}\right] .
$$

Then we call $\left[\begin{array}{ll}A_{21} & A_{23}\end{array}\right]$ a row Hankel block, and we call $\left[\begin{array}{ll}A_{12}^{T} & A_{32}^{T}\end{array}\right]^{T}$ a column Hankel block. The HSS representation exploits the lowrank structure of these types of blocks, but not all of them. It restricts itself to a fixed set hierarchical set of blocks instead. (This is to be contrasted with the SSS representation which does capture the low-rank structure of all the relevant Hankel blocks.)

To this end we need some notation. Let $A=A_{0 ; 0,0} \in \mathcal{R}^{n \times n}$, and let $n=n_{0 ; 0}$. We assume that there is a partition tree associated to $A$, which is defined as follows. We assume that for each $0 \leq k<K$ there are non-negative integers $n_{k ; i}$, for $i=0, \ldots, 2^{k}-1$, such that $n_{k ; i}=n_{k+1 ; 2 i}+n_{k+1 ; 2 i+1}$. Note that these numbers can be naturally associated with a binary tree which we call the partition tree. (In general we do not need to have a complete binary tree, but we ignore that generalization here.)

Based on this partition tree we recursively partition $A$ as follows:

$$
A_{k ; i, j}={ }_{n_{k+1 ; 2 i+1}}^{n_{k+1 ; 2 i}}\left(\begin{array}{cc}
n_{k+1 ; 2 j} & n_{k+1 ; 2 j+1} \\
A_{k+1 ; 2 i, 2 j} & A_{k+1 ; 2 i, 2 j+1} \\
A_{k+1 ; 2 i+1,2 j} & A_{k+1 ; 2 i+1,2 j+1}
\end{array}\right),
$$

and these sub-blocks can be viewed as edges on the partition tree.

We define the row Hankel blocks at each level as:

$$
\mathcal{H}_{k ; i}=\left[\begin{array}{llllll}
A_{k ; i, 0} & \cdots & A_{k ; i, i-1} & A_{k, i, i+1} & \cdots & A_{k, i, 2^{k}-1}
\end{array}\right] .
$$

That is, $\mathcal{H}_{k ; i}$ is the i'th row at the k'th level with the block $A_{k ; i, i}$ deleted. In analogous manner, one can define column Hankel blocks $\mathcal{V}_{k ; i}$, where the block columns of $A$ at the $k$-th level are considered instead. Next let the matrix $V_{k ; i}$ be such that its columns form a basis for the row space of $\mathcal{H}_{k ; i}$. Similarly let the matrix $U_{k ; i}$ be such that its columns form a basis for the column space of $\mathcal{H}_{k ; i}$.

Since $\mathcal{H}_{k, i}$ shares sub-matrices with $\mathcal{H}_{k+1 ; 2 i}$ and $\mathcal{H}_{k+1 ; 2 i+1}$, it follows that there are translation matrices $R_{k ; i}$ such that

$$
U_{k ; i}=\left[\begin{array}{c}
U_{k+1 ; 2 i} R_{k+1 ; 2 i} \\
U_{k+1 ; 2 i+1} R_{k+1 ; 2 i+1}
\end{array}\right] .
$$

Similarly there are translation matrices $W_{k ; i}$ associated with $V_{k ; i}$. We define the expansion coefficients $B_{k ; i, j}$ as follows:

$$
\begin{aligned}
& A_{k ; 2 i, 2 i+1}=U_{k ; 2 i} B_{k ; 2 i, 2 i+1} V_{k ; 2 i+1}^{T} \\
& A_{k ; 2 i+1,2 i}=U_{k ; 2 i+1} B_{k ; 2 i+1,2 i} V_{k ; 2 i}^{T},
\end{aligned}
$$

for $0<k \leq K$. We also define

$$
A_{K ; i, i}=D_{K ; i} .
$$

We next observe that the translation matrices can be much smaller than the basis matrices. Therefore in the HSS representation we store only $U_{K ; i}$ and $V_{K ; i}$. To recover the other basis matrices we use $R_{k ; i}$ and $W_{: k ; i}$ instead. Therefore the complete HSS representation of $A$ is the partition tree $n_{k ; i}$ along with the leaf-level matrices $D_{K ; i}, U_{K ; i}, V_{K ; i}$, the translation matrices $R_{k ; i}, W_{k ; i}$, and the expansion coefficients $B_{k ; 2 i, 2 i+1}, B_{k ; 2 i+1,2 i}$. With this we can check that 
every entry of $A$ can be uniquely recovered from the HSS representation.

If $p_{k ; i}$ denotes the rank of $\mathcal{H}_{k ; i}$ and $q_{k ; i}$ the rank of $\mathcal{V}_{k ; i}$, then it can be verified, for example, that $R_{k+1 ; 2 i} \in \mathcal{R}^{p_{k+1 ; 2 i} \times p_{k ; i}}$ and $B_{k ; 2 i, 2 i+1} \in \mathcal{R}^{p_{k ; 2 i} \times q_{k ; 2 i+1}}$. So the ranks of the row and column Hankel blocks determines how small the HSS representation is.

Just as in the SSS case, there exists an $O\left(n^{2}\right)$ algorithm to construct an optimal HSS representation directly from the entries of the matrix [5]. However, in special cases we can do much better. For sparse matrices the construction can be done in linear time [5]. For matrices of the form $A_{i, j}=f\left(x_{i}, y_{j}\right)$, where $f: \mathcal{R}^{d} \rightarrow \mathcal{R}$ the FMM techniques of Greengard and Rokhlin can be used to compute the HSS representation of $A$ in linear time provided $f$ satisfies some nice properties [18]. However, in practice the HSS representation is most often computed quickly from the fact that matrix algebra in HSS form can be done quickly.

Just as in the SSS case, the key observation is that there is a short HSS representation as long as the row and column Hankel blocks have small rank. Then one observes for example, that the sum of two HSS matrices will have column (row) Hankel block ranks that are the sum of the corresponding column (row) Hankel blocks of the summands. Therefore it is not surprising that there is a linear time algorithm to add two HSS matrices.

The superfast multiplication of two HSS matrices is based on the following observation:

$$
\begin{aligned}
& {\left[\begin{array}{lll}
A_{11} & A_{12} & A_{13} \\
A_{21} & A_{22} & A_{23} \\
A_{31} & A_{32} & A_{33}
\end{array}\right]\left[\begin{array}{lll}
B_{11} & B_{12} & B_{13} \\
B_{21} & B_{22} & B_{23} \\
B_{31} & B_{32} & B_{33}
\end{array}\right]=} \\
& {\left[\begin{array}{ccc}
* & * B_{12}+A_{12} *+* B_{32} & * \\
A_{21} *+* B_{21}+A_{23} * & * & A_{21} *+* B_{23}+A_{23} * \\
* & * B_{12}+A_{32} *+* B_{32} & *
\end{array}\right],}
\end{aligned}
$$

where $*$ 's denote matrices whose ranks are irrelevant. From this we see that the ranks of the column (row) Hankel blocks of the product are the sums of the ranks of the corresponding colum (row) Hankel blocks of the multiplicands. Again there is a linear time algorithm to compute the HSS representation of the product from the HSS representation of the multiplicands.

To give a flavor of how HSS algorithms are constructed we go back to the problem of fast matrix-vector multiplications $A x=b$, where $A$ is in HSS form. We will use the notation $x_{K ; i}$ to denote the $i$-block of $x$ when it is partitioned according to the cuts at the leaf level of the partition tree. Then with a little bit of algebra one can show that the following recursions will do the job:

$$
\begin{aligned}
g_{K ; i} & =V_{K ; i}^{T} x_{K ; i} \\
g_{k ; i} & =W_{k+1 ; 2 i}^{T} g_{k+1: 2 i}+W_{k+1 ; 2 i+1}^{T} g_{k+1 ; 2 i+1}, \quad k<K, \\
f_{0 ; 0} & =[] \\
f_{k ; 2 i} & =R_{k ; 2 i} f_{k-1 ; i}+B_{k ; 2 i, 2 i+1} g_{k ; 2 i+1}, \quad 0<k \leq K, \\
f_{k ; 2 i+1} & =R_{k ; 2 i+1} f_{k-1 ; i}+B_{k ; 2 i+1,2 i} g_{k ; 2 i}, \quad 0<k \leq K, \\
b_{K ; i} & =D_{K ; i} x_{K ; i}+U_{K ; i} f_{K ; i} .
\end{aligned}
$$

It is a clear that this leads to a linear time algorithm. Just like in the SSS case these recursions can also be used to give a diagonal representation of $A$ and to also give a linear time solver for computing $x$ given $b$ via sparse Gaussian elimination.
Towards this define the pair, $Z_{\downarrow}$ and $Z_{\leftrightarrow}$, of linear operators on the binary partition tree, via the equations:

$$
\begin{aligned}
\left(Z_{\downarrow} x\right)_{k ; i} & =x_{k-1 ;\left\lfloor\frac{i}{2}\right\rfloor} \\
\left(Z_{\leftrightarrow} x\right)_{k ; 2 i} & =x_{k ; 2 i+1} \\
\left(Z_{\leftrightarrow} x\right)_{k ; 2 i+1} & =x_{k ; 2 i} .
\end{aligned}
$$

Also define the linear projection operator $P$ on the binary partition tree such that $P^{T} g$ restricts $g$ just to the leaves of the tree, $\left(P^{T} g\right)_{i}=$ $g_{K ; i}$.

As before also define the block diagonal matrices $D, U$ and $V$, which only consist of the entries $D_{K ; i}, U_{K ; i}$ and $V_{K ; i}$ that are on the leaves of the binary partition tree. Also define the block diagonal matrices $R$ and $W$ that consist of the entries $R_{k ; i}$ and $W_{k ; i}$ which are defined on all nodes of the binary partition tree. Finally define the block diagonal matrix $B$ which consists of the entries $B_{k ; 2 i, 2 i+1}$ and $B_{k ; 2 i+1,2 i}$ by making a natural association with the nodes of the binary tree. Similarly we will use $g$ to denote a column vector containing all the $g_{k ; i}$ 's.

Then we can re-write the fast recursions for the multiplication $A x$ as

$$
\begin{aligned}
g & =Z_{\downarrow}^{T} W^{T} g+P V^{T} x \\
f & =R Z_{\downarrow} f+B Z_{\leftrightarrow} g \\
b & =D x+U P^{T} f .
\end{aligned}
$$

In matrix form this appears as:

$$
\left[\begin{array}{ccc}
I-Z_{\downarrow}^{T} W^{T} & 0 & -P V^{T} \\
-B Z_{\leftrightarrow} & I-R Z_{\downarrow} & 0 \\
0 & U P^{T} & D
\end{array}\right]\left[\begin{array}{l}
g \\
f \\
x
\end{array}\right]=\left[\begin{array}{l}
0 \\
0 \\
b
\end{array}\right] .
$$

This implies that the HSS diagonal representation of a matrix $A$ is given by

$$
A=D+U P^{T}\left(I-R Z_{\downarrow}\right)^{-1} B Z_{\leftrightarrow}\left(I-Z_{\downarrow}^{T} W^{T}\right)^{-1} P V^{T},
$$

which also shows that $A$ is just the Schur complement of a larger block sparse matrix with the binary partition tree with edges only between siblings, as the incidence graph.

Since such a binary partition tree has a fill-in free elimination order, this also shows that we can get a linear time solver for constructing $x$ given $b$, by first re-ordering the unknowns (and the equations) in the order: $\left(g_{K ; i}, f_{K ; i}, x_{K ; i}\right)$, followed by $\left(g_{k ; i}, f_{k ; i}\right)$.

If numerical stability is a concern, it is also clear that a linear time sparse $Q R$ factorization algorithm can also be constructed by similar considerations.

If $A$ has a short HSS representation then so does $A^{-1}$ and this can be computed in linear time from that of $A$. To see how and why this is possible, first observe that:

$$
\begin{gathered}
{\left[\begin{array}{ccc}
A_{11} & A_{12} & A_{13} \\
A_{21} & A_{22} & A_{23} \\
A_{31} & A_{32} & A_{33}
\end{array}\right]=} \\
{\left[\begin{array}{ccc}
I & 0 & 0 \\
A_{21} & I & 0 \\
* & \left(A_{32}+* A_{12}\right) * & I
\end{array}\right]\left[\begin{array}{ccc}
* & A_{12} & * \\
0 & * & A_{23}+A_{21} * \\
0 & 0 & *
\end{array}\right],}
\end{gathered}
$$

where again $*$ 's denote matrices whose ranks are irrelevant. From this it follows that the $L U$ factors of $A$ will have the same column 
and row Hankel block ranks as that of $A$ itself. Furthermore, with a little bit bit of algebra, a set of fast linear time recursions can be worked out for computing the HSS representations of the $L U$ factors from that of $A$.

From:

$$
\left[\begin{array}{ccc}
U_{11} & U_{12} & U_{13} \\
0 & U_{22} & U_{23} \\
0 & 0 & U_{33}
\end{array}\right]^{-1}=\left[\begin{array}{ccc}
* & * U_{12} & * \\
0 & * & * U_{23} * \\
0 & 0 & *
\end{array}\right],
$$

we observe that the columns and row Hankel blocks of the inverse of a triangular matrix have the same ranks as those of the original matrix. Furthermore a linear time algorithm can be devised for computing the HSS representation of the inverse of a triangular HSS matrix.

We already know that the product of two HSS matrices will have short HSS representations, but the Hankel block ranks will add up. So when we multiply $U^{-1} L^{-1}=A^{-1}$ there is the danger that we will end up with larger column and row Hankel block ranks. However, we note that:

$$
\begin{aligned}
& {\left[\begin{array}{lll}
A_{11} & A_{12} & A_{13} \\
A_{21} & A_{22} & A_{23} \\
A_{31} & A_{32} & A_{33}
\end{array}\right]^{-1}=} \\
& {\left[\begin{array}{ccc}
* & \left(* A_{12}+* A_{32}\right) S_{v} & * \\
S_{h}\left(A_{21} *+A_{23} *\right) & * & S_{h}\left(A_{21} *+A_{23} *\right) \\
* & \left(* A_{12}+* A_{32}\right) S_{v} & *
\end{array}\right],}
\end{aligned}
$$

which shows that the column and row Hankel block ranks of $A^{-1}$ are at most that of $A$ itself. Therefore with a little algebra we can devise linear time algorithms to compute the HSS representation of $A^{-1}$ from those of the $L U$ factors of $A$. The above formula is also the reason for the definition of row and column Hankel blocks.

\section{CONCLUSION}

We have presented a basic outline of the displacement structured matrices and the construction of fast solvers for them. However the biggest open question in this area is whether this approach generalizes to dealing with Toeplitz-block-Toeplitz (TBT) matrices?

Currently the most efficient solvers convert Toeplitz matrices to Cauchy-like matrices via the FFT and exploit their HSS structure instead. However, even this approach does not seem to extend to TBT matrices, though in general the HSS approach does not do too badly if there is an underlying 2D kernel function (via the FMM representation).

For SSS and HSS type representations the biggest open question is the exact rank structure of the inverse of discrete 2D Laplace like matrices. There are several papers on the approximate low-rank structure in special cases, but the general question remains quite open.

We have made some remarks recently on this problem [4]. Our key observation is to note that the complexity of SSS algorithms depends critically on the underlying linear graph, while that of HSS depends on the special binary partition tree. Conversely the lack of a fast direct solver for FMM representations can be traced to the difficulty of doing Gaussian elimination quickly on a the more complicated FMM tree. This immediately raises the issue of tying the graph structure more intimately to the matrix representation and the associated fast algorithms.
This problem is important as our understanding of the inverse of discrete 3D Laplace like matrices is not sharp enough to yield fast enough practical solvers and the underlying graph is the $3 \mathrm{D}$ mesh.

\section{REFERENCES}

[1] Alin Bostan, Claude-Pierre Jeannerod, Christophe Mouilleron, and Éric Schost. On matrices with displacement structure: generalized operators and faster algorithms. CoRR, abs/1703.03734, 2017.

[2] Shivkumar Chandrasekaran, Patrick Dewilde, Ming Gu, William Lyons, and Timothy Pals. A fast solver for hss representations via sparse matrices. SIAM fournal on Matrix Analysis and Applications, 29(1):67-81, 2006.

[3] Shivkumar Chandrasekaran, Patrick Dewilde, Ming Gu, T Pals, Xiaorui Sun, Alle-Jan van der Veen, and Daniel White. Some fast algorithms for sequentially semiseparable representations. SIAM fournal on Matrix Analysis and Applications, 27(2):341-364, 2005.

[4] Shivkumar Chandrasekaran and Nithin Govindarajan. The exact fine structure of the inverse of discrete elliptic operators. In SIAM Conference on Applied Linear Algebra (SIAM-ALA18), 2018.

[5] Shivkumar Chandrasekaran, Ming Gu, and Timothy Pals. A fast ulv decomposition solver for hierarchically semiseparable representations. SIAM fournal on Matrix Analysis and Applications, 28(3):603-622, 2006.

[6] Shivkumar Chandrasekaran, Ming Gu, X Sun, J Xia, and J Zhu. A superfast algorithm for toeplitz systems of linear equations. SIAM fournal on Matrix Analysis and Applications, 29(4):1247-1266, 2007.

[7] Shivkumar Chandrasekaran, Ming Gu, Jianlin Xia, and Jiang Zhu. A fast qr algorithm for companion matrices. In Recent advances in matrix and operator theory, pages 111-143. Springer, 2007.

[8] Shivkumar Chandrasekaran and Ali H Sayed. Stabilizing the generalized schur algorithm. SIAM fournal on Matrix Analysis and Applications, 17(4):950-983, 1996.

[9] J Chun and T Kailath. A constructive proof of the gohberg-semencul formula. Linear Algebra and its Applications, 121:475-489, 1989.

[10] James W Cooley and John W Tukey. An algorithm for the machine calculation of complex fourier series. Mathematics of computation, 19(90):297-301, 1965.

[11] Patric Dewilde and Alle-Jan van der Veen. Time-Varying Systems and Computations. 011998

[12] Patrick Dewilde and Shivkumar Chandrasekaran. A hierarchical semi-separable moore-penrose equation solver. In Wavelets, multiscale systems and hypercomplex analysis, pages 69-85. Springer, 2006.

[13] Yuli Eidelman, Israel Gohberg, and Iulian Haimovici. Separable type representations of matrices and fast algorithms. Springer, 2014.

[14] Joachim von zur Gathen and Jurgen Gerhard. Modern computer algebra. Cambridge University Press, Cambridge, 1999.

[15] Israel Gohberg and Vadim Olshevsky. Fast algorithms with preprocessing for matrix-vector multiplication problems. Fournal of Complexity, 1994.

[16] Israel Gohberg and A. Semencul. On the inversion of finite toeplitz matrices and their continuous analogs. Mat. Issled, 1972.

[17] Gene H Golub and Charles F Van Loan. Matrix computations. JHU Press, 4 edition, 2013.

[18] Leslie Greengard and Vladimir Rokhlin. A fast algorithm for particle simulations. Journal of computational physics, 73(2):325-348, 1987.

[19] Wolfgang Hackbusch. Hierarchical matrices: algorithms and analysis, volume 49. Springer, 2015.

[20] G Heinig and V Olshevsky. The schur algorithm for matrices with hessenberg displacement structure. Contemporary Mathematics, 281:3-16, 2001.

[21] Thomas Kailath, Sun-Yuan Kung, and Martin Morf. Displacement ranks of matrices and linear equations. Journal of Mathematical Analysis and Applications, 68(2):395-407, 1979.

[22] Thomas Kailath and Ali H Sayed. Displacement structure: theory and applications. SIAM review, 37(3):297-386, 1995.

[23] K Lessel, M Hartman, and Shivkumar Chandrasekaran. A fast memory efficient construction algorithm for hierarchically semi-separable representations. SIAM fournal on Matrix Analysis and Applications, 37(1):338-353, 2016.

[24] William Lyons. Fast algorithms with applications to PDEs. $\mathrm{PhD}$ thesis, University of California, Santa Barbara, 2005.

[25] Timothy Pals. Multipole for Scattering Computations: Spectral Discretization,Stabilization, Fast Solvers. PhD thesis, University of California, Santa Barbara, 2004

[26] Vladimir Rokhlin. Rapid solution of integral equations of classical potential theory. Fournal of computational physics, 60(2):187-207, 1985

[27] Vladimir Rokhlin. Rapid solution of integral equations of scattering theory in two dimensions. Fournal of Computational Physics, 86(2):414-439, 1990. 
[28] Zhifeng Sheng, Patrick Dewilde, and Shivkumar Chandrasekaran. Algorithms to solve hierarchically semi-separable systems. In System theory, the Schur algorithm and multidimensional analysis, pages 255-294. Springer, 2007.

[29] Paige Starr. On the numerical solution of one-dimensional integral and differential equations. PhD thesis, Yale University, 1992.

[30] Jianlin Xia, Yuanzhe Xi, and Ming Gu. A superfast structured solver for toeplitz linear systems via randomized sampling. SIAM fournal on Matrix Analysis and Applications, 33(3):837-858, 2012 